\title{
Masculinidades y relaciones de género en universitarios. Facultad de Ciencias Sociales. UNMSM, 2015
}

Recibido: 07/10/2016

Aprobado: 14/11/2016

\author{
Pricila León Pretel \\ Universidad Nacional Mayor de San Marcos \\ < pricilalp@hotmail.com > \\ Alejandro Choque Martínez \\ Universidad Nacional Mayor de San Marcos \\ < aschoque@yahoo.com >
}

\begin{abstract}
RESUMEN
El presente estudio se propone describir los rasgos de masculinidades que presentan los jóvenes universitarios en la Facultad de Ciencias Sociales de la Universidad Nacional Mayor de San Marcos. Los conceptos que guían la investigación son los expuestos principalmente por R.W. Connel (1995), P. Bourdieu (1990) y M. Kimmel (1994). La metodología es cualitativa: se aplicó un cuestionario diseñado con la escala de Likert para poder identificar la intensidad de convicciones en cada afirmación. Participaron 40 estudiantes de las Escuelas Académico Profesionales de Sociología, Antropología e Historia, cuyas edades fluctúan entre los 20 y 23 años. Los principales resultados nos muestran que los universitarios presentan algunos elementos de masculinidades emergentes o alternativas; manteniéndose aún en ellos los elementos principales de la masculinidad hegemónica, como: autoridad, poder y dominio de la esfera pública. Sin embargo, el perfil que los describe estaría ya influenciado por la formación académica que tienen los participantes, formación que incluye cursos sobre género tema que es transversal al proyecto educativo diseńado para todas las especialidades de la facultad.
\end{abstract}

Palabras Clave: masculinidades, masculinidad emergente, masculinidad hegemónica, relaciones de género, estudiantes universitarios.

\section{Masculinities and gender relationships in university students. Faculty of social sciences. UNMSM, 2015}

\begin{abstract}
This study describes the types of masculinities presented by young university students in the Faculty of Social Sciences at San Marcos University. The concepts guiding the research are mainly three, exposed by RW. Connel (1995), P. Bourdieu (1990) and M. Kimmel (1994). The methodology is qualitative; we applied a questionnaire designed with the Likert scale to identify the intensity of belief in each statement. Forty students from the Academic Professional Schools of Sociology, Anthropology and History participated, their ages range from 20 to 23 years old. The main results show that the university students have some elements of emerging masculinities; keeping the main elements of hegemonic masculinity, such as: authority, power and dominion of the public sphere. However, the profile that describes them would already be influenced by participants' academic training which includes training courses on gender issue that is transverse to the educational project designed for all specialties of the faculty.
\end{abstract}

KEYwORDs: Masculinities, emerging masculinity, hegemonic masculinity, gender relationships, university students. 


\section{Introducción}

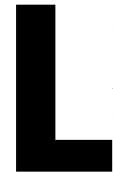

as ciencias sociales estudian las relaciones de producción y las que se erigen, a su vez, sobre ellas; totalidad que condiciona, asimismo, el desarrollo de las poblaciones y grupos sociales. Esta idea de los hechos sociales nos permite entender de manera integral un fenómeno como el estudiado. Sobre esta relación entre los hombres, Marx (1980[1859]) señala:

En la producción social de su existencia, establecen determinadas relaciones, necesarias e independientes de su voluntad, relaciones de producción que corresponden a un determinado estadio evolutivo de sus fuerzas productivas materiales. La totalidad de ellas constituye la estructura económica de la sociedad, la base real sobre la cual se alza un edificio jurídico y político, y a la cual corresponden determinadas formas de conciencia social (p. 4).

Los objetivos de esta investigación se dirigen a conocer si las masculinidades del sector de estudiantes consultado se acercan más al modelo emergente o al modelo hegemónico, cada uno de ellos vinculados, por múltiples relaciones, a las clases y mentalidades existentes. Nos interesa conocer qué elementos de este último modelo se mantienen y cuáles están en un proceso de cambio, encaminados a una relación de género más igualitaria. El incremento de la violencia contra la mujer torna, más necesario aún, desarrollar mayores estudios que profundicen, desde distintas perspectivas, el problema planteado, que contribuyan a definir políticas de estado adecuadas al logro de una solución integral.

Actualmente el conflicto de género es uno de los problemas más preocupante para nuestra sociedad, entre los años 2013-2015 ocurrieron 302 muertes por feminicidio y 504 tentativas por la misma razón. La edad del $60 \%$ de los agresores fluctúa entre 18 y 35 años. La primera causa identificada son los celos; los agresores, en un 73\%, han sido pareja o ex pareja de la víctima. Según el diario Perú 21 (8,10-2014) nuestro país ocupa el segundo lugar en feminicidio en América Latina. Nos preguntamos: ¿cuáles son las causas sociales que convierten a un hombre en asesino de su propia pareja? En la búsqueda de una respuesta al problema, uno de los aspectos explorados lo constituye el estudio de las masculinidades existentes y el impacto que puedan tener en estos hechos funestos.

En este trabajo nos interesa conocer la masculinidad existente en un sector de alumnos de CCSS, cuya particularidad es tener como tema de trabajo formativo la explicación y atención de los problemas de la sociedad peruana en sus múltiples relaciones. Esta investigación es parte de un trabajo mayor. Que se interesa en saber las características de la masculinidad, las formas que cobra en la variante hegemónica y la alternativa, y el contenido de clase que expresan. Así, nuestra investigación sobre el medio universitario apunta a entender un problema mayor y a brindar aportes a la solución del mismo.

En el Perú aún son insuficientes los estudios sobre masculinidades. Esta insuficiencia puede explicarse tal vez porque el problema no yace en el concepto mismo de masculinidad, sino en su uso (Machado, 2016: 81). Existen algunas instituciones como IMPARES, Universidad Cayetano Heredia, Universidad Católica del Perú y Universidad Nacional Mayor de San Marcos, que tienen algunos estudios por parte de sus docentes y alumnos. Las instituciones del Estado como el Ministerio de la Mujer y Desarrollo Social. Programa Nacional Contra la Violencia Familiar y Sexual. Intervención profesional frente al feminicidio (2012) dan cuenta de algunas causas que conllevan al feminicidio, Viviano (2012) señala como las principales causas: celos del agresor, infidelidad de la mujer, negativa de ella a tener relaciones sexuales y decisión de terminar la relación con el agresor, asimismo afirma:

Los casos de feminicidio y tentativas también se desarrollan en contextos en los que no ha existido relación sentimental presentándose una fijación obsesiva del agresor. $10 \%$ de las víctimas de feminicidio y tentativas atendidas en los CEM se había negado a ser pareja de su agresor. El 43\% de los casos mencionados como "otros" están vinculados a otras situaciones de desafíos del poder masculino. (p. 36)

El mencionado Programa también señala que los agresores son principalmente agricultores, policías, vigilantes, taxistas y moto taxistas. La mayor cantidad de feminicidios se registran en Lima Metropolitana, en distritos más poblados y más pobres como; San Juan de Lurigancho, Villa María del Triunfo, Ate, entre otros. Mapa de pobreza INEI (2014) se trata de personas con un nivel educativo básico y bajos ingresos. Estas evidencias nos sugieren que la pobreza se convierte, también, en uno de los factores de riesgo para la violencia familiar.

En este contexto de violencia familiar que vive el país son los varones, señalados y sancionados como 
agresores, pero poco se explora sobre la realidad que viven para conocer las condiciones que los condujeron a tan lamentables situaciones. Existen pocas investigaciones que nos muestran las causas sociales de estos comportamientos agresivos hacia las mujeres, que en su mayoría han tenido alguna relación de intimidad con ellos. Inclusive sobre las mujeres que sufren violencia también existen limitados estudios orientados a conocer el contexto familiar donde ocurren hechos de violencia, sus relaciones de pareja, etc. Se prioriza la asistencia social como víctimas o se estudia la manera en que son maltratadas, pero no se estudian las condiciones de vida que envuelven a estas familias protagonistas de violencia familiar.

Lo real es que los hombres, de muy diversas formas, y en condiciones sociales distintas, son educados para desempeńar roles acordes con el sistema y con su propia realidad social. Esta educación genera un tipo de expresión afectiva que está limitada igualmente por prescripciones sociales (Olarte \& de Keijzer, 2016: 35). El ejercicio del poder y la autoridad como elementos principales de la masculinidad hegemónica están presentes en sus condiciones de vida, se respiran diariamente. El elemento económico también juega un papel preponderante: por un lado el varón, irrealizado, limitado económicamente e impotente ante su situación, descarga sus frustraciones contra la mujer y los niños; la mujer, igualmente en condiciones donde no cuenta con ingreso alguno ni preparación para desempeñarse, tolera el maltrato porque no encuentra otra solución.

Nuestro estudio se dirige a los jóvenes universitarios, pues ellos poseen una formación integrada por diversos conocimientos propios de las ciencias sociales, donde los estudios de género se imparten, directa o indirectamente, en todas las especialidades. Estos jóvenes aspiran a un futuro más esperanzador para ellos y para su familia. Hemos querido conocer, pues, si sus masculinidades están alejándose de la masculinidad hegemónica, sustentada por Bourdieu y Kimmel, aquí se encuentran elementos que están relacionados con la violencia de género. En este estudio solo se ha tomado en cuenta los siguientes:

a) Subvaloración de la mujer en cuanto a su debilidad, por lo que debe ser protegida por el varón.

b) Roles domésticos en familia.

c) Homofobia.

c) Maltrato por parte del varón.

\section{Avance en los estudios sobre el tema}

El interés por estudiar las masculinidades tiene como precedentes el develamiento de la desigualdad entre el varón y la mujer iniciado por el socialismo en lucha por la emancipación de la mujer, asimismo el movimiento feminista internacional. Este último aparece desarrollado especialmente desde los años 70 del siglo XX en Occidente. Dicho movimiento exige la igualdad de derechos, cuestiona tanto la dominación masculina, como la violencia contra las mujeres, rechaza la asignación de roles sociales según el sexo por considerarla arbitraria. De este modo, los estudios sobre masculinidad van a ser preocupación de diversas disciplinas, propiamente en la década de los 80 del siglo pasado. Sin embargo, desde los 70 ya existían estudios, especialmente en Psicología y Psiquiatría, sobre este tema.

En este período se destacan los estudios sociológicos iniciados en los países anglosajones, como Australia, Reino Unido, Canadá y EE.UU. El nombre que le dieron sus autores a este conjunto de investigaciones sobre masculinidad fue Men's Studies (Estudios de hombres) El planteamiento común como resultado de dichos trabajos se resume en que no hay una masculinidad universal: existen masculinidades que varían de acuerdo al tiempo y al lugar. Kimmel (1997) ofrece la siguiente definición:

La virilidad no es estática ni atemporal, es histórica; no es la manifestación de una esencia interior, es construida socialmente; no sube a la conciencia desde nuestros componentes biológicos; es creada en la cultura. La virilidad significa cosas diferentes en diferentes épocas para diferentes personas (p. 49).

Este mismo sociólogo realizó una investigación sobre la historia de la masculinidad en Gran Bretańa y confirmó que aun en los mismos lugares y momentos las masculinidades no eran iguales. Estos hallazgos demuestran que la masculinidad es una construcción social, que no es resultante biológica, se vincula a la edad, clase social, incluso al tipo de trabajo. Para Jociles (2001) los mencionados Men's Studies tienen un enfoque constructivista social o cultural, lo que los lleva siempre a sostener que las múltiples masculinidades se definen socialmente y siempre en relación al género femenino. Es decir, los hombres son distintos a las mujeres y así tienen que formarse y convencer a la sociedad de ello. 
De aquí que cada cultura y formación social tenga su propia forma de construir la masculinidad o masculinidades de sus ciudadanos. Se puede establecer así una conclusión: la masculinidad se construye negando lo femenino. El peor insulto para un hombre suele ser decirle que se comporta como mujer. Jociles aclara, en la obra citada, que la teoría de la construcción de la masculinidad negando lo femenino no es contribución de los Men's Studies, pues ya había sido elaborada por Erik Erikson en la década de los 50 y posteriormente desarrollada por otros estudios con enfoque pos Freudiano. Aquí encontramos el gran problema. ¿La construcción de las masculinidades podrá cambiar en un sistema que reproduce permanentemente, en todas las esferas de sociedad, la dominación masculina? No se puede enseñar en una dirección y pedir que la práctica sea distinta a lo enseñado; no se puede aspirar a otros resultados cuando las condiciones donde se forman las masculinidades no cambian significativamente. En nuestro país, especialmente en los sectores más pobres, los hombres se enfrentan a condiciones de socialización muy difíciles para moldear su masculinidad, aquí la violencia es un factor que también se vive y se aprende lamentablemente.

Uno de los principales exponentes de la sociología que tratan el tema es Pierre Bourdieu, quien en su libro La dominación masculina dice que existe una visión androcéntrica que toma como natural el orden de las cosas y de las prácticas sociales que realiza el varón:

La visión dominante de la división sexual se expresa en discursos como los refranes, proverbios, enigmas, Cantos, poemas o en representaciones gráficas como las decoraciones murales, los adornos de la cerámica o de los tejidos... Si esta división parece "natural", como se dice a veces para hablar de lo que es normal, al punto de volverse inevitable, se debe a que se presenta, en el estado objetivado, en el mundo social y también en el estado incorporado, en los habitas, como un sistema de categorías de percepción, pensamiento y acción. (1990: 15-16)

De aquí podemos concluir que la masculinidad y sus características sociales son impuestas al hombre por el propio sistema y se muestra como un conjunto de prácticas sociales que han sido aprendidas y aceptadas en su medio. Para Artińano (2015) existe una masculinidad imperante, predominantemente en las sociedades de Occidente cuyas características son: Superioridad del hombre, heterosexual, blanco, de origen europeo, burgués y de religión Judeo Cristiana. Visto de esta forma la variedad de masculinidades que existen tienen en común algunos de estos elementos que se imponen por el propio sistema cultural, también de unas culturas a otras mediante la colonización como ocurrió en el Perú con la llegada de los españoles; terminamos señalando que en nuestros países como América Latina no sólo se impone las reglas económicas sino también la cultura dominante.

Los estudios de masculinidad que existen en América Latina en su mayoría se sustentan en Connel, Kimmel y Bourdieu; enfatizando que en la región las masculinidades están en crisis, pues el sistema capitalista viene limitando cada vez más a los varones el cumplimiento de sus roles tradicionales, para los que siguen siendo formados en la familia, la escuela, la religión, los medios de comunicación, etc. Existen algunas masculinidades "alternativas" pero se sigue imponiendo aún la masculinidad hegemónica. Giddens (2007) en su obra Un mundo desbocado afirma que la globalización influye de modo determinante en nuestras vidas, que nos encontramos en una sociedad con muchos riesgos e incertidumbres. El matrimonio, la sexualidad y la familia no están exentos de dichos efectos. Las presiones y tensiones están cambiando la familia tradicional.

Para Kimmel (1992) los diferentes estudios y aportes teóricos que se han venido realizando sobre los estudios de la masculinidad u hombres son una respuesta a la ambivalencia sobre la cuestión del poder que existe en la vida de los hombres, por lo cual "la mayoría de ellos se sienten existencialmente impotentes” (p.130).

Las investigaciones indican que se sienten entrampados en los viejos roles y sin tener capacidad para realizar los cambios en las relaciones sociales contemporáneas. Es quizás por eso que el movimiento feminista inició los estudios sobre masculinidades, buscando cuáles eran las características sociales y culturales que hacían al hombre tener y ejercer poder.

Sobre el feminismo, Mariátegui (2008[1924]) escribió que el feminismo de entonces era producto de una civilización en una época concreta; este nace en el Perú de las condiciones sociales que viven las mujeres que han llegado a las aulas universitarias y a un sindicato en sus centros laborales. En estos espacios desarrollan su conciencia crítica respecto a sus derechos; además que existen feminismos tales como: 
Aparte de este feminismo espontáneo y orgánico, que recluta sus adherentes entre las diversas categorías del trabajo femenino, existe aquí, como en otras partes, un feminismo de diletantes un poco pedante y otro poco mundane. Las feministas de este rango convierten el feminismo en un simple ejercicio literario, en un mero deporte de moda... la clase diferencia a los individuos más que el sexo. (2008: 1-2)

Esta investigación es de tipo descriptiva y aplicada sobre las masculinidades de los jóvenes universitarios de la facultad de Ciencias Sociales. Se ha seleccionado a los alumnos de estas especialidades dado que su quehacer académico está más directamente relacionado con el estudio de esta problemática, suponen mayor información sobre el caso.

El universo de la investigación realizada está compuesto por 128 alumnos, varones ( 43 de historia, $42 \mathrm{de}$ sociología y 43 de antropología), tercer año, quinto ciclo. La muestra seleccionada la constituyen 40 jóvenes de las Escuelas Académico Profesionales de Sociología, Antropología e Historia, elegida al azahar. Asimismo, la unidad de análisis está dada por cada joven matriculado en el semestre 2015-II que tenga un rango de edad entre 18 y 25 años.

Por lo indicado, la recolección de los datos que fundamentan la investigación procede de fuentes esencialmente primarias, de tipo cualitativo. Como instrumentos de recolección se ha utilizado la entrevista y el cuestionario. La información ha sido procesada con SPSS, v. 22.

Los hallazgos que se señalan a continuación corresponden a las respuestas ofrecidas al cuestionario aplicado, donde se exploran aspectos de la jerarquía y poder que el varón busca establecer en su relación con la mujer; la percepción que él tiene de la masculinidad, y la visión sobre la capacidad y autosuficiencia de la mujer.

\section{De los resultados}

De acuerdo al modelo de masculinidad hegemónica los roles se asignan según los sexos, así a la mujer le corresponde los roles domésticos porque ella está ubicada en la esfera privada y el varón en la pública. Veamos en la Tabla 1 esta característica en relación al grupo estudiado.

El contundente $89.48 \%$ donde los participantes rechazan la afirmación que las actividades domésticas
TABLA 1

ROLES E INDEPENDENCIA DE LA MUJER RESPECTO AL VARÓN

\begin{tabular}{llc}
\multicolumn{1}{c}{ ITEM } & $\begin{array}{c}\text { Acuerdo } \\
\text { y muy de } \\
\text { acuerdo }\end{array}$ & $\begin{array}{c}\text { Desacuerdo y muy en } \\
\text { desacuerdo }\end{array}$ \\
\hline $\begin{array}{l}\text { 1. Las actividades } \\
\text { domésticas no han } \\
\text { sido ni son asignadas } \\
\text { a los varones, porque } \\
\text { estas son propias de } \\
\text { las mujeres }\end{array}$ & & \\
\hline $\begin{array}{l}\text { 2. La mujer debe res- } \\
\text { petar al varón porque } \\
\text { eso está definido por } \\
\text { la sociedad }\end{array}$ & \multicolumn{1}{c}{$7.89 \%$} & \\
\hline $\begin{array}{l}\text { 3. La seguridad de } \\
\text { la mujer depende } \\
\text { naturalmente de su } \\
\text { pareja }\end{array}$ & & \\
\hline
\end{tabular}

Fuente: Elaboración propia

son propias de las mujeres nos sorprende gratamente; esto lo podemos atribuir a varios factores como: a) la etapa de juventud b) el ambiente académico crítico que propugna la igualdad no solo entre el varón y la mujer, sino a nivel de toda la sociedad c) la situación económica de las familias del nivel social " $\mathrm{C}$ " de donde proceden los estudiantes. En este tipo de familias es usual que ambos padres trabajen, por tanto las tareas del hogar son compartidas, el varón no se puede sustraer a esta responsabilidad. Pareciera que la forma de masculinidad construida a partir de la idea central de que la mujer es inferior no es afectada significativamente con el desempeño de los roles domésticos; mientras el varón pueda dominar a la mujer por otros medios simbólicos o prácticos. Debemos considerar, respecto a la masculinidad, lo que señala Fuller (1997) que la masculinidad es un proceso, no existe un tiempo determinado para su construcción esta va cambiando o modificando sus elementos constitutivos, en parte o en su totalidad; en algunas circunstancias se marcan unos elementos más que otros, tal es así que:

A medida que los jóvenes maduran e ingresan a la universidad o al mundo del trabajo, adquieren confianza en sí mismos y sus representaciones de masculinidad se alejan gradualmente de los ideales viriles para enfatizar la responsabilidad, el logro y el altruismo social. Es decir, dejan de ser machos para convertirse en hombres ingresando así al período de la hombría. (p.4) 
Sobre las respuestas, dos y tres de la Tabla 1, el porcentaje sumado revela también una posición avanzada de cuestionamiento a la idea tradicional de mujer, pero, como se advierte en el punto dos, se mantienen notorios rezagos de una posición de masculinidad hegemónica. Podría ser que esta idea retrógrada ya no exista en las actuales relaciones entre los jóvenes o, también que la afirmación no fue adecuada. Consideramos que sobre la seguridad, deberá precisarse, en nuevas investigaciones asignando un claro sentido de ella: económica, protección, asistencia, etc.

En cuanto a la violencia de los varones contra las mujeres y a la percepción que tienen los jóvenes participantes sobre la responsabilidad de la mujer frente al maltrato veamos la tabla 2 .

TABLA 2

VIOLENCIA DE GÉNERO CONTRA LA MUJER

$\begin{array}{lll}\text { ITEM } & \begin{array}{l}\text { Acuerdo y muy } \\ \text { de acuerdo esacuerdo y muy }\end{array} \\ \end{array}$

Las mujeres son mal-

tratadas porque ellas lo $\quad 50 \% \quad 47.37 \%$

permiten

Fuente: Elaboración propia.

Estos resultados merecen una explicación más detallada, debido a que los datos hallados en las respuestas entrañan un matiz de masculinidad hegemónica que aparentemente contradicen las respuestas claras que se registran en la Tabla 1.

De acuerdo al concepto de masculinidad, entendido como un proceso dinámico, podríamos asumir que no existe contradicción; los datos sí son coherentes con dicho concepto entendiendo que no todos los elementos de la masculinidad cambian al mismo tiempo, a veces predominan algunos. En este caso parece que la violencia contra la mujer es un factor tolerado por los estudiantes, porque la libertad e independencia que están poniendo en práctica las mujeres resiente a los hombres de algún modo. Para Menendez 2014 el hombre no ha acompańado en ese proceso de cambio, actualmente quiere una mujer que trabaje que sea independiente pero en otros aspectos que no desafíe su poder; la infidelidad o el rechazo de la mujer son hechos que pueden destruirlos emocionalmente. Como vemos no es posible encontrar un perfil de masculinidad totalmente hegemónica, tampoco totalmente distinta a esta, en el proceso de construcción afloran algunos elementos más que otros. Perder el poder y control sobre la mujer son elementos difíciles de aceptar aun por la mayoría de varones en sociedades como la nuestra. Lo real es que los hombres y por tanto su masculinidad cambian cómo cambia la historia Kimmel (1994: 2).

Nos preocupa las respuestas opuestas y polarizadas de estos dos grupos; nos interesa la mayoría que prácticamente culpa a la propia mujer de su maltrato, o sea desconocen los factores socioeconómicos y culturales, y el mismo contexto que la hacen vulnerable ante un varón, desconocen que no se trata de un enfrentamiento para ver quien sale airoso(sa) sino de un complejo proceso que afecta el respeto de los derechos de la mujer quien es maltratada o termina asesinada como ocurre cada día. La respuesta mayoritaria encubre el carácter histórico social y cultural del problema; desestima la particularidad que tiene la masculinidad y feminidad construida en el Perú, con el sustento de la familia patriarcal y patrilineal, con la fuerte influencia de la mentalidad oligárquico feudal en proceso de superación.

En la masculinidad hegemónica rasgos como el poder, el dominio y la autoridad del varón se construyen en oposición a los rasgos femeninos, pero a costa de afectar a la mujer; la construcción de uno no es posible imaginarla sin la otra, ambas construcciones son simultáneas. El que maltrata lo hace porque considera "natural" esta relación; las mujeres que asumen este rol construido son proclives a la aceptación de la violencia psicológica, sexual, física; imaginan que es algo natural en el perfil de un varón y que los excesos pueden ser atenuados o tolerados. Bourdieu describe cómo el mismo sistema se encarga de grabar en las mentes de hombres y mujeres estas formas de relación desigual entre ambos:

Debido a que se encuentra inscrito y en las divisiones del mundo social, o más concretamente en las relaciones sociales de dominio y explotación que se han instituido entre los sexos, y en las mentes, bajo la forma de los principios de división que conducen a clasificar todas las cosas del mundo y todas las prácticas según distinciones reducibles a la oposición entre lo masculino y lo femenino (Bourdieu 1990:16)

En la mayoría de los casos no es un simple problema de ejercer la voluntad, pues se trata de que la sociedad ha impedido o debilitado la construcción de esta voluntad en la mujer y la ha condicionado al extremo de hacerla 
víctima permanente de la violencia. De este modo la violencia simbólica ejercida naturaliza estos rasgos de la masculinidad hegemónica y los impone en los procesos de socialización; reproduciéndolos, cada vez con formas más sutiles, pero siempre lesivas.

El 47.37 de la Tabla 2 que rechaza la percepción de que las mujeres son maltratadas porque ellas lo permiten es un porcentaje significativo que vislumbra masculinidades con relaciones de género más equitativas.

Estas diferencias confirman que las masculinidades se construyen de forma distinta, hasta en un mismo lugar. Porque influyen varios factores como: la familia, el modelo paterno, la condición social. Los estudiantes sanmarquinos proceden, en alto porcentaje, de los estratos C y D, existiendo un promedio de $20 \%$ del D; este dato lo obtenemos a partir de los servicios sociales que brinda la universidad en las distintas facultades.

TABLA 3

MASCULINIDAD Y HOMOFOBIA

\begin{tabular}{lcc}
\hline ITEM & $\begin{array}{l}\text { Acuerdo y muy } \\
\text { de acuerdo }\end{array}$ & $\begin{array}{l}\text { Desacuer do } \\
\text { y muy en des- } \\
\text { acuerdo }\end{array}$ \\
\hline $\begin{array}{l}\text { La homosexualidad es una } \\
\text { tendencia creciente en la } \\
\text { actualidad que desvaloriza la } \\
\text { condición masculina }\end{array}$ & $26.32 \%$ & $71.05 \%$ \\
\hline $\begin{array}{l}\text { Es necesario defender la unión } \\
\text { civil , porque es el primer paso } \\
\text { para extender el derecho de } \\
\text { las parejas homosexuales al } \\
\text { matrimonio }\end{array}$ & $45.95 \%$ & \\
\hline
\end{tabular}

Fuente: Elaboración propia

La homofobia es un elemento que caracteriza a la masculinidad hegemónica; los tres autores que hemos tomado como importantes para esta investigación concuerdan en este aspecto. Las masculinidades en general se construyen en oposición a la feminidad, es una ofensa para cualquier hombre que lo comparen con una mujer, desde que son púberes ya se enfrentan entre pares para demostrar que son hombres y no mujeres.

En la Tabla 3 se plantea la relación masculinidad hegemónica y homosexualidad. Se explora si esta última llevaría a desvalorizar a la primera. Las respuestas halladas registran resultados claros en cuanto a los límites que se establece en esta relación. Un notorio 26\% admite que desvaloriza la masculinidad, la afecta; en tanto que un significativo $71,05 \%$ opina lo contrario.

Se expresa en este sector de la juventud una mentalidad más tolerante y comprensiva de esta realidad social, pero también se evidencia un fuerte rezago aún de rechazo. Como se ha señalado aquí, creemos que el contexto académico que viven los estudiantes es un factor importante a tener en cuenta para interpretar estos datos. El ambiente universitario es propicio para cuestionar y criticar muchos conceptos, hechos y aspectos de la cultura tradicional que en otro momento, anterior o posterior, pueden o podrán verse en forma distinta. Por ejemplo, las creencias religiosas, inculcadas por la familia y el sistema, se ven objetadas en este período.

Igualmente, la no aceptación de la unión civil, entendida como paso previo al matrimonio homosexual, expresada por el $50 \%$ establece una polarización que nos indica, en primer lugar, los límites claros que pone este sector a la concepción de familia, y al rol que desempeñan el hombre y la mujer en este espacio. Sin embargo vemos como un $50 \%$ sí aceptaría el matrimonio de parejas homosexuales considerándolo un derecho de todos. Queda por explorar las razones que conducen a una o a otra. Con la información obtenida, no es posible establecer cómo se desenvolvería esta aceptación en los casos que afectaran a una parte de la propia familia. El tema, así, plantea muchas interrogantes que permitirían explorar las direcciones en la que se va forjando esta nueva percepción.

\begin{tabular}{|c|c|}
\hline ITEMS & PORCENTAJES \\
\hline Ocuparse de las tareas cotidianas & $31 \%$ \\
\hline Trabajar y traer dinero a la casa & $26 \%$ \\
\hline Ser la autoridad y dirigir la familia & $25 \%$ \\
\hline Administrar la economía de la familia & $18 \%$ \\
\hline
\end{tabular}

Fuente: Elaboración propia

En la Tabla 4, en cuanto a los roles del hogar preferido por los estudiantes, encontramos que los de proveedor, y autoridad de la familia, que apuntan más claramente en la dirección de cualidades de la masculinidad hegemónica relacionadas con el poder, están identificados por los estudiantes como roles propios de los varones, incluso administrar la economía del hogar se suma a este concepto de masculinidad.

Estos tres roles preferidos, en teoría, por los estudiantes están vinculados al modelo hegemónico de masculinidad; sin embargo el problema surge cuando 
tienen que asumir estas responsabilidades en la prácti$\mathrm{ca}$, en contextos histórico sociales diversos. Desde hace aproximadamente tres décadas el sistema familiar en el Perú ha entrado en crisis y con ello la masculinidad hegemónica. Este sistema encontró su fundamento económico social en la revolución industrial, la cual contribuyó a dejar atrás la familia de la etapa feudal y sus sistemas internos de autoridad, donde el varón es el patriarca, autoridad y guía de la familia nuclear; dedicado a la producción en el ámbito público y la mujer dedicada a la reproducción cuidadora de los niños y el hogar, Olavarría (2003) además afirma:

Ello fue acompañado de una legislación, especialmente tomada del derecho civil napoleónico, que le daba la autoridad al varón dentro de la familia y exigía obediencia de la mujer, y de una organización del trabajo que permitiría a los hombres ser proveedores principales o únicos del hogar mientras las mujeres criaban y cuidaban de sus hijos y hogar, aunque esto último fue en muchos casos más un discurso ideológico que un logro efectivo. (p. 92)

La crisis de la familia actual consiste en que el nuevo contexto neoliberal no garantiza que los varones puedan ostentar la masculinidad hegemónica sustentada básicamente en el poder económico. El trabajo se ha precarizado, sin derechos sociales, con ingresos cada vez más disminuidos, en muchos casos la tecnología han desplazado la mano del hombre. En estas condiciones, las mujeres se han volcado al mercado a buscar ingresos para complementar los de su pareja o para sustentar a su familia en la ausencia de ésta; esta sería una causa fundamental de crisis para la familia actual. (Olavarría 2003).

Las respuestas de los estudiantes reflejan un ideal alejado de la realidad, o en todo caso complicado para su cumplimiento debido básicamente al sistema imperante y por ello justamente más sentida por las mayorías de nuestro país; no es casual que la violencia y los feminicidios ocurran en los lugares mayormente donde han mayor pobreza.

El mundo de la vida doméstica siempre fue simbólicamente asignado a la mujer como si se tratara de una obligación natural. La cocina, hasta antes del éxito de la gastronomía peruana, era mayoritariamente relacionado con los roles de la mujer. Las respuestas Consolidadas en la Tabla 5 tienen interesantes novedades.
TABLA 5 ACTIVIDADES REALIZADAS EN EL TIEMPO LIBRE

Si tuviera sólo cuatro horas libres a la semana qué actividad sería principal para usted

\begin{tabular}{|l|r|}
\hline & Porcentaje \\
\hline 1. Descansar & $30.24 \%$ \\
\hline 2. Hacer deporte & $18.60 \%$ \\
\hline 3. Cocinar para su pareja o familia & $18.60 \%$ \\
\hline 4. Salir con sus amigos/as & $16.28 \%$ \\
\hline 5. Lavar su ropa y ordenar su casa & $13.95 \%$ \\
\hline 6. No opina & $2.33 \%$ \\
\hline Total & $100 \%$ \\
\hline
\end{tabular}

Fuente: Elaboración propia

Las tareas domésticas (3 y 5) tienen un 32.55\% de preferencia, lo que indica un viraje que se va produciendo, de un tercio, en este sector de la juventud desde las posiciones de masculinidad hegemónica patriarcales a una más democrática y equitativa, respuestas coherentes con las de la tabla 1. Este proceso se opera a medida que se desarrolla el capitalismo en el Perú, pero lleva el hallazgo de las limitaciones del mismo donde se persista en la lucha por lograr una sociedad más justa, realmente democrática y moderna. El descanso y el deporte tienen un espacio significativo aquí, aunque es materia a explorar si estas actividades lo vinculan afectuosamente con la pareja. Como se observa, los estudiantes mayormente prefieren actividades que marcan la diferencia con las actividades femeninas, pero sigue la tendencia a ocuparse también de actividades que la sociedad sigue considerando como propias de la mujer.

Los resultados que arrojan la Tabla 6 van también en la dirección de definir el nuevo perfil de la juventud.

TABLA 6

PERFIL DE LA MUJER IDEAL

\begin{tabular}{|l|c|}
\hline İTEMS & Porcentaje \\
\hline 1. Ser inteligente & $40.28 \%$ \\
\hline 2. Estar de acuerdo con sus decisiones & $20.78 \%$ \\
\hline 3. Bella físicamente & $15.58 \%$ \\
\hline 4. Esmerada en los cuidados con usted & $10.39 \%$ \\
\hline 5. Que se desempeñe bien en el hogar & $7.79 \%$ \\
\hline 6. No opina & $5.19 \%$ \\
\hline TOTAL: & $100 \%$ \\
\hline
\end{tabular}

Fuente: Elaboración propia 
Aquí pareciera que la belleza física (1.), como atractivo de la mujer, no fuera importante; quizás los estudiantes tengan ese ideal que claramente expresa una admiración por la inteligencia de la mujer (2.), porque están compartiendo tareas que hacen visible esta cualidad femenina. En las características de la mujer ideal, algunas alternativas como "estar de acuerdo con sus decisiones" (2.), "esmerada en los cuidados con usted"(4.) y "que se desempeñe bien en el hogar" (5.) suman un significativo 39\%. Lo que indica que los participantes sienten que ellos deben decidir como jefe de familia, pues por ser varones les corresponde el poder para ello y la mujer debe aceptar sus decisiones; siendo una de las tareas de éstas el cuidado de los varones. En realidad la dominación del varón es muy velada, a veces totalmente invisible por las mujeres y por toda la sociedad.

\section{Conclusiones}

Los estudiantes están transitando a masculinidades alternativas que expresan una mayor valoración de la mujer en cuanto a su independencia, libertad, inteligencia; no tan sometida a los roles tradicionales. Reconocen que ellos deben participar en los roles del hogar, que aún siguen considerando propios de la mujer. Sin embargo, se muestran los datos donde un $25 \%$, aproximadamente, presentan aún los rasgos más visibles de masculinidades tradicionales, es decir hegemónicas.

El contexto en que se encuentran los estudiantes, sobre todo la esfera académica influye en el desarrollo de sus tipos de masculinidades. Principalmente por el espíritu crítico, de análisis y de cambio respecto a la realidad social; además los participantes no proceden de familias pobres extremas, donde las masculinidades podrían estar más identificadas con la masculinidad hegemónica.

La construcción de las masculinidades no es ajena al problema de clases sociales, a sus formas de relaciones y a las vivencias cotidianas de acuerdo a su ubicación. La violencia se puede dar en cualquier hogar, pero donde se exacerba y cobra vidas diariamente es en los sectores más pobres de la sociedad. La crisis familiar y la crisis de la masculinidad, tanto la de mentalidad oligárquica como la de entrańa burguesa, en sus formas más visibles se da en un contexto de precariedad económica y social; aquí el varón de clase em- pobrecida encuentra muy difícil cumplir sobre todo con su rol de proveedor, es decir, el que se ocupa de atender todas las necesidades materiales de la familia; contrariamente sigue siendo formado culturalmente para ello, esta es la mayor contradicción que tiene que afrontar en la realidad. La mujer también, particularmente la que no ha tenido oportunidad de capacitarse para enfrentar retos económicos, sigue viendo en el varón a un proveedor y protector. Si bien el cuestionamiento a formas de masculinidad más visiblemente opresivas se desarrolla en el escenario social, convocando inclusive movilizaciones masivas. Otras, más sutiles, se vigorizan en el país.

\section{Referencias bibliográficas}

Artiñano, Néstor (2015). Masculinidades incómodas: jóvenes, género y pobreza. Editorial Espacio. Buenos Aires.

Bourdieu, Pierre (1996). La dominación masculina. Editorial Anagrama. Barcelona.

Bourdieu, Pierre \& Passeron, J. C. (2001). Fundamentos de una teoría de la violencia simbólica. En La reproducción. Elementos para una teoría del sistema de enseñanza, 13-85.

Connel, Robert W. (1997). La organización social de la masculinidad. En Masculinidadles: poder y crisis, Cap. 2, ISIS-FLACSO: Ediciones de las Mujeres, $\mathrm{N}^{\circ}$ 24, 31-48.

Fuller, Norma (1997). Fronteras y retos: varones de clase media del Perú. En Masculinidadles: poder y crisis. Ediciones de las Mujeres, (24).

Giddens, Anthony (2007). Un mundo desbocado, los efectos de la globalización en nuestras vidas. México:Taurus.

Instituto Nacional de Estadística e Informática (2014) Mapa de pobreza.

Jociles, María Isabel (2001). El estudio sobre las masculinidades. Panorámica general. En Gazeta de Antropología, 12. España. Universidad de Granada.

Kimmel, Michael (1997). Homofobia, temor, vergüenza y silencio en la identidad masculina. En Masculinidadles: poder y crisis, Cap. 3, ISIS-FLACSO: Ediciones de las Mujeres, $\mathrm{N}^{\circ} 24,49-62$.

Kimmel, Michael (1992). La producción teórica sobre la masculinidad: nuevos aportes. En e. Regina Rodríguez (Ed.), Fin de siglo: Género y cambio civilizatorio. Isis Internacional. Recuperado el 10 de junio de 2015, de http:// www.popularesydiversas.org/media/uploads/documentos/investigacion/subtema_1_genero_y_cambio_civilizatorio.pdf. 
Machado, Yanela (2016). Aproximación a la comprensión de las masculinidades. Sistematización de autores europeos. En Revista Sexología y Sociedad, 22(1), 68 -86.

Mariátegui, José Carlos (2008). Marxists Internet Archive. Recuperado de: https://www.marxists.org/espanol/tematica/index.htm

MarX, Karl (1980). Contribución a la crítica de la economía política. Siglo XXI.

Menéndez Cóndor, Pamela Fabiola (2014). Transformaciones de las prácticas amorosas de los jóvenes de las nuevas clases medias y populares de Lima Norte. Tesis para optar el grado de Licenciada en Sociología, UNMSM.

Olarte, Carlos Arturo \& de Keijzer, Benno (2016). El rostro universitario de la afectividad: una perspectiva desde los varones. En Perspectivas docentes, 26(59), 30-39.
Olavarría, José (2003). Los estudios sobre masculinidades en América Latina. Un punto de vista. Anuario Social y Politico de América Latina y El Caribe, 6.

OlavarRía, José (2001). Y todos querían ser (buenos) padres. FLACSO. Santiago, Chile

Ruiz-Bravo, P. (2015). Desde el margen. Representaciones de la masculinidad en la narrativa joven en el Perú. Revista de Estudios de Género. La Ventana, 2(12), 244-271.

Viveros Vigoya, Mara (1997). Los estudios sobre lo masculino en américa latina. Una producción teórica emergente.

VIVIANo, Teresa (2012). Intervención profesional frente al feminicidio. Aportes desde los CEM para la atención y prevención. MIMP, Lima. 